International Journal of Biological Sciences

ISSN 1449-2288 www.biolsci.org 2006 2(4):186-187

Letter to the editor

(C)2006 Ivyspring International Publisher. All rights reserved

\title{
The new portrait of mammary gland stem cells
}

\section{Rui-Hong Wang}

Genetics of Development and Diseases Branch, National Institute of Diabetes and Digestive and Kidney Diseases, National Institutes of Health, 10/9N105, 10 Center Drive, Bethesda, MD 20892, USA.

Correspondence to: Rui-Hong Wang, Tel: (301) 496-5445, Fax: (301) 480-1135, e-mail: Rui-HongW@intra.niddk.nih.gov

Received: 2006.04.24; Accepted: 2006.05.23; Published: 2006.06.10

Breast cancer occurs at rate of 1 out of 7 . After so many years of research, even though treatment has been dramatically improved and survival rate and length significantly increased, there is still not cure for the disease. The possible existence of mammary gland stem cell and breast cancer stem cell [1,2] lights the hope for finding a cure to this deadly disease. The rational behind is that once the stem cell can be isolated, the signalling pathways that regulate its proliferation, self-renewal, survival and differentiation will be illustrated, and this might shed light on the mechanisms of breast cancer formation, therefore leading to a better therapeutic treatment.

Existence of stem cell in normal mammary gland has been convincingly demonstrated by Kordon and Smith [1]. In their report, an entire mammary gland could be regenerated with the progeny of a single cell following transplantation into cleared mammary fat pads. By definition, mammary gland stem cells are those that rarely divide and persist throughout reproductive life. Classical markers [3] for identifying and purifying mammary stem cells are label retention (tritiated thymidine or BrdU), stem cell antigen Sca-1 expression, ability to exclude dyes such as Hoechst 33342 or rhodamine 123 (side population -- SP, e.g. Hoechst 33342 negative) because of elevated expression of membrane transporter proteins, such as P-glycoproteins, and "small light" cell by electronic microscopy. However, these profiling methods are controversial and confusing. Sometimes, for a layman, it is very difficult to handle.

But this situation is about to change with two recent publications in Nature. In January, Shackleton et al published "Generation of a functional mammary gland from a single stem cell" [4]. In this report, the authors cleared mammary gland mixture with CD31 (endothelial marker), CD45 and TER119 (haematopoietic antigens) by FACS sorting ( $\mathrm{Lin}^{-}$ population). Using repopulating cleared mammary fat pad (Mammary repopulating 'units'--MRUs) as criteria, they were able to increase the MRUs from $1 / 4900$ to $1 / 64$ just by applying two more markers on Lin population -- CD29 (beta1-integrin) and CD24 (heat-stable antigen). Lin-CD29hiCd24+ ${ }^{+}$cells have expended differentiation ability and colony-formation ability. A single Lin-CD29hiCD24+ cell can repopulate cleared mammary fat pad and develop into a fully functioning mammary gland, demonstrating its high proliferating and multi-lineage differentiation capacity in vivo. Lin-CD29hiCd24+ cells can self-renew. In mammary gland of MMTV-Wnt-1 transgenic mice, Lin-CD29hiCd $24^{+}$population are increased and mammary gland outgrowths of Lin-CD29hi Cd24+ MMTV-Wnt-1 cells are profoundly hyperplastic. LinCD29hiCd24+ cells were enriched for long-term labelretaining cells, CD49f $\mathrm{f}^{+}$cells. However, neither high Sca-1 expression nor Hoechst33342 dye exclusion was enriched in this population.

In the February publication "Purification and unique properties of mammary epithelial stem cells" [5], Stingl et al purified CD45-Ter119-CD31CD49fhiCD24med cells and demonstrated that they were the mammary gland stem cells. In consistency, CD45-Ter119-CD31-CD49fhiCD24 ${ }^{\text {med }}$ cells were Sca-1 negative and only minority of these cells can efflux Hoechst 33342 and Rhodamine-123. Interestingly, the authors took one step further to illustrate that CD45Ter119-CD31-CD49fhiCD24med cells are in G1 or S/G2/M fractions, indicating the stem cell population is a cycling population.

Most notably, these two publications completely changed the old mammary gland stem cell picture -Hoechst 33342 negative, slowly dividing and Sca-1 positive. They demonstrated that CD45-Ter119-CD31CD49fhiCD24med and Lin-CD29hiCd24+ are the mammary stem cell populations, whereas previous SP and Sca- $1^{+}$cells only take very few percentage of these two populations if not at all. Since label retention coincides very well with $\mathrm{Lin}-\mathrm{CD} 2 \mathrm{Chigh}^{\mathrm{C}} \mathrm{CD}^{24+}$ or $\mathrm{CD} 45^{-}$ Ter119-CD31-CD49fhicD24med, it joins CD29, CD49f and CD24 as one of the most efficient 4 mammary gland stem cell markers. These new markers make it easier to isolate mammary gland stem cells, therefore open a door for further characterizing these cells. Importantly, with the same markers, cancer stem cells can be purified as well. This provides a new opportunity to develop new targeted therapies to killing cancer stem cells. Finally, the report proved that mammary gland stem cells were actually cycling within cell cycle. This observation lays an important foundation for testing new methods of chemoprevention and chemotherapy. 


\section{References}

1. Kordon EC, Smith GH. An entire functional mammary gland may comprise the progeny from a single cell. Development 1998; 125:1921-30.

2. Al-Hajj M, Wicha M, Benito-Hernandez A et al. Prospective identification of tumorigenic breast cancer cells. PNAS. 2003; 100:3983-8.

3. Kalirai H, Clarke RB. Human breast epithelial stem cells and their regulation. J Pathol. 2006; 208: 7-16.

4. Shackleton M, Vaillant F, Simpson KJ et al. Generation of a functional mammary gland from a single stem cell. Nature 2006; 439:84-8.

5. Stingl J, Eirew P, Ricketson I et al. Purification and unique properties of mammary epithelial stem cells. Nature 2006; 439:993-7. 